\title{
(อ) OPEN ACCESS \\ Soldiering on: a survey on the lived experience of tinnitus in aged military veterans in the UK
}

\author{
Georgina Burns-O'Connell, ${ }^{1,2}$ David Stockdale, ${ }^{1}$ Derek James Hoare ${ }^{2,3}$
}

'British Tinnitus Association, Sheffield, UK

${ }^{2}$ Department of Hearing Sciences, Division of Clinical Neuroscience, School of Medicine, University of Nottingham, Nottingham, UK ${ }^{3}$ NIHR Nottingham Biomedical Research Centre, University of Nottingham, Nottingham, UK

\section{Correspondence to}

Dr Georgina Burns-O'Connell, Research Officer, British Tinnitus Association, Sheffield S8 OTB, UK; georgina@tinnitus.org.uk

Accepted 22 July 2019 Published Online First 24 September 2019

\section{Check for updates}

(c) Author(s) (or their employer(s)) 2019. Re-use permitted under CC BY-NC. No commercial re-use. See rights and permissions. Published by BMJ.

\section{To cite: Burns-}

O'Connell G, Stockdale D,

Hoare DJ. Med Humanit

2019:45:408-415.

\section{ABSTRACT}

Tinnitus has long been interrogated as a medical conundrum, with little discourse between medicine and other disciplines. It involves the perception of sound in the ears or head without any external sound source, most likely a natural consequence of some form of hearing loss. For many people, tinnitus is bothersome and associated with various problems such as insomnia, difficulty concentrating and impaired listening ability. Nevertheless, with little attention from humanities or the social sciences, our understanding of the wider perspectives and psychosocial context of adults with tinnitus is limited, especially among UK military veterans. The aim of this study was to explore the impact of tinnitus on aged UK veterans, and to consider the support they receive and require to live well with tinnitus. In all, 120 aged UK veterans took part in this study. Data revealed similarities and differences between UK veteran and other study populations. For example, tinnitus symptom severity was higher in aged veterans than a general (younger) research population, particularly so on measures of intrusiveness and the effect of tinnitus on listening ability. Veterans had mixed views on social support. Many did not want to talk about tinnitus with others and/or did not want to burden their family, preferring to deal with their tinnitus 'backstage'. Others appreciated empathy or sympathy; many implied a desire that their family and/or friends could better understand their experience of living with tinnitus and the problems it caused them. These complexities support a need for cross-disciplinary work to understand and respond to tinnitus-related problems in veterans.

\section{INTRODUCTION}

Tinnitus (the experience of sound within the ear, ears or head, without any external source) has long been pondered by the medical profession. ${ }^{1}$ There is no objective measure to determine tinnitus presence and most often it remains medically unexplained. In most cases, tinnitus is subjective, in that the sound can only be heard by the individual reporting it. Despite or perhaps because of its enigmatic nature, little research to date has been cross-disciplinary. Yet, tinnitus is multi-factorial, causing a plethora of human difficulties that need to be more fully understood and countered. ${ }^{2}$

Ageing and noise exposure represent commonly appreciated risk factors for tinnitus and so particular populations such as aged military veterans likely represent high-risk groups. Independently, research on tinnitus and research on military veteran populations have been extensive in recent years, and conducted within many disciplines. For example, there has been a number of studies which have reviewed veterans' needs ${ }^{3}{ }^{4}$ and their access to healthcare services. ${ }^{5-7}$ Recently, there has been an interest in the heterogeneous nature of tinnitus ${ }^{8}$ and the different problematic domains of tinnitus experienced, ${ }^{9}$ and some qualitative exploration relating to patients' experiences of treatments/interventions. ${ }^{10} 11$

However, there is relatively little qualitative research on the experience of tinnitus in the veteran population. In Lost Voices, ${ }^{12}$ the Director General of The Royal British Legion refers to hearing damage (and by implication tinnitus), as one of the less visible effects of warfare, raising concern that it has received very little attention in a UK context to date. In contrast, there has been considerable investment in US research on military noise-induced hearing loss, identifying that one in four service members returning from conflicts complain of hearing loss and/or tinnitus. ${ }^{13}$ It is reasonable to assume parallels exist between US and UK ex-service personnel. The Lost Voices report calls for 'epidemiological research (to) be commissioned and published on the prevalence and impact of tinnitus on UK military personnel, to ascertain the scale of the problem'. It also recommended that the "National Institute for Health and Care Excellence (NICE) develops guidelines on best practice treatment and support for patients with tinnitus' and that 'a clear pathway to access support for tinnitus within the MOD [Ministry of Defence] referral pathway is established'. A further report (Call to Mind) from Forces in mind (2015) highlights the need for research to explore whether it would be more effective to integrate veteran support into general services or to provide veteran-specific ('culturally sensitive') services. While NICE are now developing broad clinical practice guidance on tinnitus, other recommendations have yet to receive attention. As such, there is a significant gap in research on tinnitus in UK veterans.

Most previous research in veteran populations has been conducted in the USA where research is embedded within a Veteran Affairs hospital. Much of this research has either tested interventions or explored quantitative relationships between predefined variables. ${ }^{14}$ However, no research study has yet taken a qualitative approach to explore the lived experience of veterans with tinnitus. There is also little reported in the existing tinnitus literature on the demographic characteristics (eg, age, gender, ethnicity, or job role and type/level of occupational noise exposure) of ex-service personnel.

The aim of this study was to explore the impact of tinnitus on aged veterans and the support they 
receive and require to live well with tinnitus, from a shared medical and social scientific perspectives.

\section{METHODS}

This study is reported according to the Survey Reporting Guideline. ${ }^{15}$ The study was cross-sectional, and involved a questionnaire completed by veterans with tinnitus who were born before first January 1950 (a requirement of the project funder). We chose the method of survey for this first study to allow rapid collection of the relatively large-scale data that would give a sense of how generalisable individual experiences and problem domains were among veterans. This study was reviewed and given favourable opinion by the University of Nottingham Faculty of Medicine \& Health Sciences Research Ethics Committee and the University of Nottingham was study sponsor.

\section{Questionnaire}

Tinnitus symptom severity was measured using the Tinnitus Functional Index (TFI), ${ }^{16}$ a composite, self-report questionnaire. The TFI shows high internal consistency $(\alpha=0.80)$, extremely high test-retest reliability (Intraclass Correlation Coefficient $=0.86$ ), borderline test-retest agreement (93\%) and good convergent validity with other tinnitus questionnaires $(>0.8) .{ }^{17}$ The TFI contains 25 items and eight subscales. Each item is scored by the participant on a 10 -point scale, and the total score is divided by 2.5 to give a global score out of 100 .

To explore views and needs with respect to tinnitus, a brief additional questionnaire was developed. Initial items were derived by the research team, and critically reviewed for readability and face validity by members of the Patient and Public Involvement panel at the NIHR Nottingham Biomedical Research Centre (hearing theme). The final version of the questionnaire included six open-ended questions:

- As well as tinnitus what conditions/illnesses (physical or mental health) have you been diagnosed with?

-What do you think caused your tinnitus?

- What healthcare/treatment or other forms of support for tinnitus have you accessed?

- Thinking specifically about your tinnitus, in what ways is it a problem to you?

- What support do you have for your tinnitus from those around you (eg, family and/or friends), and what do you find useful?

- What healthcare/treatment or other form of support do you think would be especially helpful for you and other veterans?

\section{Sample selection}

Participants were individuals born before 1 January 1950 who were residing permanently in the UK and who served at least 1 day in Her Majesty's Armed Forces (British Armed Forces). This included surviving World War two veterans, those who undertook National Service, and other voluntary enlisted veterans.

Given an estimated UK population of 300000 veterans have hearing loss, a conservative estimate of aged veterans with tinnitus is in the region of 60000 (one-third or all aged veterans). ${ }^{18}$ For a $95 \%$ confidence interval and an assumed $10 \%$ margin of error (eg, in TFI scores), we required at least 96 participants. However, we specified a closing date for survey completion and actively promoted the study until that date to maximise recruitment.

\section{Survey administration}

The survey was launched in August 2018 and closed to participants in December 2018. The main mode of administration was online, via SurveyMonkey (www.surveymonkey.com). A paper version of the questionnaire was also made available by post. Invitations to participate were disseminated through many channels including social media, British Tinnitus Association media and information days and events, and via The Royal British Legion, other veteran charity networks, and the Medical Advisory Committee. Participants were informed about the intent of the research and made aware of their right to withdraw, and that completion of the questionnaire was taken as consent for the anonymised data provided to be used in the research and dissemination. No financial incentives were provided.

\section{Analysis}

The study only required a single point of participation to complete a questionnaire either online or on paper. A response was taken to be the completion of the TFI and/or at least one of the open questions. To be a valid dataset, it was required that participants answered at least 19 of the 25 items in the TFI. For open-ended questions, partial data were acceptable. Descriptive statistics (demographics, including TFI scores) were performed in Excel. Free-text responses were analysed using a thematic analysis approach. ${ }^{19}$ Responses to the questions were most often brief, and so to avoid any misinterpretation of meaning that could occur due to a lack of context, we coded responses using only the verbatim text rather than what was implied. ${ }^{20}$ Authors independently analysed the entire dataset, this involved data familiarisation (reading and rereading) and the extraction of any meaningful codes from each response. At the same time, authors considered potential themes to represent those quotes. Themes and representative quotes were then compared across authors to agree a final set of themes that best represented all of the data. ${ }^{21}$ The authors' position was acknowledged throughout the research process and it is understood that our cultural, personal and past experiences shaped our interpretations of the data. ${ }^{22}$

${ }^{23}$ For example, authors were biassed by their different backgrounds (social science, nursing, neuroscience) and experience (of similar research in other populations) mainly in terms of the language used to describe similar or the same observations in the data. However, all discrepancies were resolved through discussion and revisiting the original data where necessary.

\section{RESULTS}

\section{Demographic of responders}

The survey was completed by 120 participants (118 men, 1 woman and 1 not reported). As this was the first such study of this population in the UK, it is difficult to determine how representative the population of respondents was. Age was reported in categories: 14 participants were aged 68-69 years; 32 were aged 70-74 years; 29 were aged $75-79$ years; 30 were aged 80-84 years; nine were aged $85-89$ years; four were aged $\geq 90$ years and one respondent did not report their age. In all, 117 participants identified their ethnicity as white UK; one Irish; one Polish and one English/American.

\section{Tinnitus scores}

The sample included participants who had a broad range of tinnitus durations: 1 year or less $(n=3) ; 2-5$ years $(n=6)$; $6-10$ years $(n=17) ; 11-20$ years $(n=25) ; 21-30$ years $(n=12)$ and over 30 years $(n=57)$. Tinnitus symptom severity ranged across almost the full range of possible TFI global scores (0-99) 


\begin{tabular}{|c|c|c|}
\hline & Mean/100 & SD \\
\hline TFI total score & 45.26 & 24.30 \\
\hline \multicolumn{3}{|l|}{ TFI subscales } \\
\hline Intrusiveness & 62.18 & 37.44 \\
\hline Sense of control & 54.09 & 30.18 \\
\hline Cognitive & 38.42 & 28.87 \\
\hline Sleep & 36.19 & 31.25 \\
\hline Auditory & 51.47 & 29.22 \\
\hline Relaxation & 50.63 & 27.12 \\
\hline Quality of life & 36.79 & 28.54 \\
\hline Emotional & 37.56 & 30.49 \\
\hline
\end{tabular}

TFI, Tinnitus Functional Index.

with an average TFI score of $45.26(\mathrm{SD}=24.30)$ (table 1). The average score is interpreted as tinnitus being a moderate problem requiring clinical intervention. ${ }^{24}$

The majority of participants had experienced and/or been diagnosed with other health conditions, in addition to experiencing tinnitus. Some of the identified conditions are commonly comorbid with tinnitus, for example, hearing loss, anxiety and depression. Other reported conditions/illnesses, for example, eyesight problems and asthma, are not commonly comorbid with tinnitus. Other conditions that may be indirectly associated with tinnitus included spinal problems, cancer and myalgic encephalomyelitis. Various age-typical conditions such as hypertension, diabetes and heart conditions, were also reported.

\section{Qualitative findings}

Data collected from the free-text questions asked at the end of the survey were analysed and organised into five themes (box 1).

\section{Perceived causes of tinnitus}

Strong themes relating to what the veterans perceived to have caused their tinnitus emerged from the data. Many of the participants attributed their tinnitus to the noise exposure they had experienced during their time serving in the armed forces. The most notable noise identified as responsible for their tinnitus was that produced by 'gunfire'. One participant recalls during his National Service training that 'after (the) first shot (I had) ringing in my right ear'

When asked what they think caused their tinnitus, others acknowledged their exposure to industrial noises from engines and machinery while serving the Royal Navy and the Royal Air Force:

Noise in the cockpit of helicopters

howling turbines in Royal Navy machinery spaces.

\section{Box 1 Qualitative themes}

Perceived causes of tinnitus

- Tinnitus-related problems experienced by aged veterans

- Aged veterans use of healthcare/treatment or other forms of support for tinnitus

- What veterans want from healthcare/treatment and other forms of support

- Social support for tinnitus: what family and friends do, and do well
A common theme acknowledged by the ex-service personnel was the lack of hearing protection equipment provided to them during their training or service in the Armed Forces. As one participant who was a 'marksman' and his role consisted of firing recalled:

there was no mention of ear defenders in the late 50 s and early 60 s and I believe my tinnitus stems from this.

Other participants identified the cause for their tinnitus as being exposed to loud noises while at work outside of their service in the military. For example:

Exposure to loud noise throughout my working life starting as an apprentice in a structural steel works where loud noise was constant all day. I then spent 4 years in the RAF and was subject to aircraft engine noise etc. On leaving the RAF I worked in various engineering environments all subject to loud noise. The last job I had was in the print industry which was the only one where ear protection was provided although this did not eliminate all noise.

While some participants were confident in their answers regarding what had caused their tinnitus, others were not able to identify a specific cause replying, 'don't know'. Situated in between these answers were those who thought there could be a combination of factors which caused their tinnitus. For example, one respondent summarised this succinctly by stating he believed his tinnitus was caused by:

exposure to loud noise at various times through life for example, work, loud traffic, live music, attending international rugby matches, using live ammunition on firing ranges during service in the Royal Air Force

\section{Tinnitus-related problems experienced by aged veterans}

The answers to the question 'in what way is tinnitus a problem to you' were organised into three themes: medical, social, and emotional problems.

Some participants used a medical description to explain how their tinnitus was a problem to them. These responses included descriptions of the experiences of how tinnitus sounded to the individual, such as:

ringing in my ears all the time

Constant hissing is always there. Occasionally becomes a low frequency whistle, which makes me aware when I have pushed the problem to the back of my mind.

For many participants, tinnitus was a problem because it acted as a barrier to them enjoying a full social life. One participant explained their tinnitus was a problem to them:

One, when going to the opera and concerts. Two, when the subject of tinnitus arises in conversation ...

Tinnitus was viewed as an obstacle to maintaining conversations with others meaning it could disrupt the individual's interactions with other people:

I have difficulty hearing what people are saying.

The constant buzzing interferes with my ability to hear conversation clearly.

An annoying distraction in many ways, especially when I'm in conversation, trying to concentrate on something, or just trying to relax.

A number of the participants described how their tinnitus impacts on their emotional well-being, saying: 
[it] has a detrimental effect on my general demeanour.

[I have] total awareness of ringing in [my] ears and trying to control the anger and frustration that it causes.

[It is] distracting / undermining / [I have]no control.

[I] have got used to it over the years but it is still very annoying.

A small number of veterans stated they were able to manage their tinnitus such that it now has little impact on their lives, for example:

I am fortunate that it does not bother me.

[It] initially affected [my] ability to sleep but with time I have adjust-

ed and can manage to ignore most of the time.

when I do not think about it, I can handle it.

Aged veteran use of healthcare/treatment or other forms of support for tinnitus

A common finding was that the ex-service personnel had accessed healthcare or treatments via the National Health Service. Many respondents named their local hospital's audiology clinic as their point of contact, and some mentioned visiting their Ear Nose and Throat department, or a hearing therapist. Some respondents had positive negative experience of tinnitus treatment with the medical professional telling them they would 'just have to put up with it'

The most common form of treatment/healthcare option the participants identified were hearing aids, with some identifying these as 'for loss of hearing' rather than as treatment for their tinnitus.

Some of the ex-service personnel had learnt to successfully manage their tinnitus with one participant saying:

I have become used to living with it and just get on with things. I have hearing aids in both ears which are a great help.

Other participants had not benefitted from successful healthcare support with one participant explaining they had tried 'everything the NHS has [to offer] which isn't much. The Tinnitus Clinic, acupuncture, hypnotherapy, hearing aids, CR modulator [commercial sound therapy], support group[s]'.

While some participants felt they had tried all available treatments and healthcare options, many of the ex-service personnel had not accessed any forms of healthcare support for their tinnitus. Some stated this was because they had either lost 'faith' that there would be any successful treatments or because they 'didn't know that there were any [and] thought that it was incurable'.

In contrast, one participant who-when describing their tinnitus—said 'it rules my life', had taken a proactive approach to their healthcare by self-referring to a hospital unit for mental health support:

After having decided to end it all, I managed to sign myself in to a psychiatric hospital and since then (12 years [ago]) I have had steady contact with [a] Dr...

There was a difference in the access to healthcare and treatment the ex-service personnel had made which had led to a variety of support and treatment being sourced.

\section{What veterans want from healthcare/treatment and other forms of support}

When asked what healthcare/treatment would be helpful, two themes were prominent in the data. The first placed emphasis on the place of technology in tinnitus treatment. Participants considered 'noise generators', 'noise cancelling hearing aids' and National Health Service funding for 'better hearing aids', could be helpful. The second theme related to information/education and support. Many participants suggested accessible local 'workshops' and 'support groups', while others suggested better access to hospital audiology departments and specialist tinnitus services was required. One participant felt that it would be helpful to have a dedicated veteran's hospital as are established in the US.

Social support for tinnitus: what family and friends do, and do well Support provided by family and friends was perceived to be minimal by some of the veterans within this study. When asked what support they receive from their family and friends, responses included:

Very little. Patience and understanding when [I'm] unable to hear is about the only thing that might help.

As there is nothing one can do about tinnitus you just try and live with the best you can. There is no real support from people around you.

There was a challenge in translating how the experience of tinnitus impacts of the individual and their everyday life, and explaining this to family and friends, meaning many of the participants felt those in their social networks had little understanding of their experience of tinnitus:

Whilst friends and family say they understand, I don't think they really do which makes things difficult at times and that's where the frustration jumps in.

It's difficult to get support from anyone because I'm the only one that can hear it.

While some ex-service personnel had difficulty in communicating their experience of tinnitus to others, some of the veterans had decided not to tell their family and friends about their tinnitus:

I have not told any of my family of the problem.

One reason for this may have been because they did not want to be deemed to be an inconvenience to their relatives or friends:

These quotes highlight the precarious ways in which those who live with tinnitus have to negotiate whether 'revealing' their experience of tinnitus to those around them would hinder, rather than help to manage the condition. Due to the complexity of the experience of living with tinnitus, many of the ex-service personnel decided to not 'bother' those around them, and, as one participant stated he would rather:

just soldier on to [the] end of the road.

\section{DISCUSSION}

\section{The influence of tinnitus on self-presentation and social interactions among ex-service personnel: applying a sociological construct to the lived experience of tinnitus}

In the current study, analysis of responses to open-ended questions about the influence tinnitus may have on the lives of aged veterans revealed a theme about the impact tinnitus had on the participants' social interactions. From a social scientist perspective, the data we observe above can be interpreted using Goffman's work 'The Presentation of Self in Everyday Life' (Erving Goffman, 1969)which focuses on micro-levels of 
social interaction between individuals and the roles assumed during interactions. Goffman explains that during interaction, at an individual level, there are 'performers' and there are 'audiences'. In dramaturgical analysis, the central concept of 'region behaviour' is identified, explaining that we often find ourselves bounded by 'front' and 'back' stage performances. Both front and back regions are defined by their relative position to the audience; front stage both performer and audience are present, while back stage is only open to the performer meaning the audience are not permitted there. The performer can 'remove their mask' or stop their performance once they are backstage without risking damage to the audience impressions which they present and manage when frontstage. Backstage is a private space where information which may have been censored during the front stage performance is acknowledged and experienced by the performer. Access to both regions are 'controlled in order to prevent the audience from seeing backstage' (Goffman 1969, p.210).

It is important to note that due to the reduced number of people present, the 'backstage region' may often be quieter than 'frontstage', meaning tinnitus levels could be perceived to be increased due to a decrease in background (distraction) sounds. Backstage is a place where the individual can be alone and can process emotion. This private space can be an important environment to facilitate and/or prepare tinnitus management. The subjective nature of how tinnitus is experienced lends itself to increased consideration by those who live with it, particularly when in their backstage capacity.

Striving to portray as 'normal' when frontstage means the aged veterans have learnt to manage performances so they do not 'reveal' their backstage, or rather, how or when they cope with their tinnitus. The veterans suggested that if their backstage was revealed to the audience, they believed it would indicate they are not coping' with their tinnitus. By keeping their experience of tinnitus backstage, the veterans could hide the real impact of their tinnitus from their family. Some veterans felt that if they 'performed' coping with their tinnitus in the front region where their 'audience' (in this case their family members) they would become aware that their tinnitus had an impact on their lives which, in turn, could make their experience of tinnitus worse. Consequently, they mostly therefore preferred to keep how they coped with their tinnitus backstage. For example, using the term 'just soldier on' could be interpreted as continuing to keep the impact that tinnitus has on their lives backstage, to give a performance of 'coping' with their experience of tinnitus when frontstage.

Avoiding social situations and interaction could compound the problems experienced by the individual living with tinnitus, and social avoidance could damage social relationships, potentially leading to potential social isolation. One participant in the current study highlighted the persistent nature of his tinnitus regardless of the setting, describing it as:

Continuous buzzing in both ears compounded in quiet surroundings, difficulty hearing when in conversation, unable to escape from the continuous noise.

Goffman $(1969,221)$ explains that the observed can influence the observer by repositioning their 'frame of reference' which, in turn, enables them to dedicate their efforts to impression management, which results in their desired impression being achieved. Some of the ex-service personnel spoke of how they decided not to attend social events and to remain in their current setting of 'backstage' (at home) to avoid a situation where noise was present and where their tinnitus could make conversations with others difficult. This prevented them from being in 'frustrating' social situations and maintained the required impression for their 'audience' or social network:

When in a loud room of people talking or loud music I am unable to hear conversation because of the sound of the tinnitus - it sounds like a washing machine filling - the louder the noise, the more the tinnitus noise is.

If the participants' partner was aware that they had tinnitus their own social life may also be impacted by their partner's preference to avoid social settings by remaining 'backstage'. Goffman $(1969,210)$ also recognised the concept of 'teams' within a given performance, saying that:

among members of the team we find that familiarity prevails, solidarity is likely to develop, and secrets that could give the show away are shared and kept.

The veteran's partner may opt to support their performance by joining the veteran in also withdrawing from social events, which could, in turn, lead them to become isolated from their own social networks. This also highlights the practical engagement a team member (ie, veteran's partner) may provide to maintain a performance. One veteran emphasised the positive effect this 'teamwork' could have of their relationships by saying:

My wife can hear a sparrow cough at 800 yards, I find that very helpful... We make a grand team.

When actors are 'frontstage', Goffman (1969) argues that they use a script to help navigate their performance in any given social setting. When talking about their tinnitus, the veterans indicated strong signs of available 'scripts' which they had available to them. These scripts were predominantly present when the participants spoke of their tinnitus in a descriptive medical context. For example, descriptions of the physical experience of tinnitus included the following: 'buzzing', 'loud screaming in my right ear', 'hissing in my ear' and 'very high pitched noise which causes a headache'. The ongoing nature of the symptom was referred to by some participants and they described it as being 'constant' and 'always there'. Furthermore, the 'lack of silence' was highlighted as a point of distress for some with one ex-service personnel stating:

I feel a fraud on remembrance day as I never have silence not even for two minutes.

The readily available explanatory medical script which some of the participants were fluent in, highlighted the lack of script available for many of the ex-service personnel when talking about how their tinnitus impacts them on a social level. This led to some participants not telling any of their family or friends about their tinnitus because:

People around you without tinnitus are unable to understand the impact it has on your life.

The aged ex-service personnel mostly lacked a script to communicate with their social networks about how their tinnitus could impact on their interactions and relationships:

because it's not a visual thing you cannot easily portray your suffering, consequentially not many, if at all, know how you feel. 
Without a known script available to them to explain their social experience of tinnitus and the barriers it could present, there was a sense that the aged veterans were inclined to avoid social settings and interactions meaning they increasingly spent more of their life 'backstage'. Those who developed a social interaction tinnitus script were more likely to explain their experience of tinnitus and how it can make everyday life interactions challenging to their family. This has potential to allow those around them to make adjustments to their interactions that enable the ex-service personnel to continue to participate in social communications and events in a healthy way.

Given the difficulties highlighted relating to social interaction and communication, an intervention that might lend itself to improved social communication has recently been evaluated in US veterans. A programme called coping effectiveness training (CET) was evaluated by comparing it to two other interventions (acceptance and commitment therapy (ACT) and cognitive behavioural therapy (CBT)), and to a no intervention control (Martz Erin, 2018). One of the few significant comparisons found that CET led to greater improvements in social support coping than the no intervention control condition. CET aims to teach individuals to identify changeable stressors and match appropriate strategies to better help people to manage their tinnitus more effectively than the more commonly used interventions which tend to mainly focus on unwanted thoughts and emotions (as is the case with ACT and CBT). More importantly, in the context of available social scripts and interactions, CET was the only intervention used to explicitly teach skills for communicating with others about the experience of living with tinnitus, as well as how to seek social support. Having the language and skills (ie, a 'script') available to discuss their experience of living with tinnitus among their social networks to encourage an empathetic response to their challenges could help veterans to establish a system of support which encourages continued time spent 'frontstage' engaging in beneficial social interactions.

Without a social interaction tinnitus script, the participants were unable to inform their social network of the impact tinnitus had on their interactions at an individual or social level. A lack of available script could make it is incomprehensible for those close to aged veterans to understand their experience of tinnitus or to understand the impact it can have on their 'role'/'performance' in everyday life. For example, the frustration and anger coping with tinnitus can provoke for someone living with tinnitus can have a detrimental impact on their interactions. Negotiating everyday life with tinnitus can have a detrimental effect socially on the individual. Living with tinnitus can make it challenging for veterans to adopt, perform and maintain a suitable 'role' which enables them to adhere with the norms and values of social interaction.

\section{The medical perspective}

This study involved the collection of data on the characteristics, experiences of tinnitus, and perspectives on tinnitus healthcare and support, of a cohort of 120 aged veterans in the UK. The characteristics of the population were notable from many perspectives. All but one participant were male, 98\% identified as being of UK origin and various comorbid conditions were reported. Tinnitus symptom severity as measured by the TFI indicated that it is, on average, a moderate problem in this population and at a level where intervention is indicated. The average TFI score (45.26 points) was lower than that overserved in a general UK clinical population (average 50.8 points), and US veteran populations (average 56.1 points). This is to be expected as the latter were actively attending audiology departments for tinnitus management. ${ }^{25}{ }^{26}$ However, it is higher than the average score observed across general research population studies in the UK (average 40.6 points). ${ }^{27}$ Although these comparisons are with populations who were, on average, younger and with a shorter duration tinnitus, they do suggest that at least some tinnitus-related problems are more a problem in veterans than in the general population. Comparison of subscale scores here, with those in Fackrell et al (2016), suggests that the intrusiveness of tinnitus and interference with listening ability are particularly problematic in veterans. This is reflected in our free-text responses where the veterans talked about the constancy of tinnitus, and its interference with social activities and enjoyment of music. Interestingly, the problems reported by veterans were generally in line with a recent study from Watts et al (2018) which identified 18 problem domains in a large general clinical population. One of the major domains in that work was Fear, including, for example, fear of the unknown, or fear that tinnitus represents something sinister. Fear-related problems were not prominent in the current dataset. This may be because of the duration of tinnitus in this population; they have had tinnitus for a long time or may be a reflection of this distinct population and their particular resilience.

Indeed, the reported duration of tinnitus in veterans is noteworthy. A majority of veterans in this study had experienced tinnitus for more than 20 years, which far exceed that typically reported in general population studies. One caveat to this, however, is that the population that were all aged over 68 years. This was a requirement of the funder and, even though the aged make up $60 \%$ of UK veterans, limits the generalisability of the findings somewhat; if younger veterans were included, this would certainly have changed the distributions of tinnitus duration and other variables.

Across general population studies, there is also significant evidence that tinnitus symptom severity improves over time, that is, individuals develop coping strategies and adapt to tinnitus. ${ }^{28}$ Taken together, it is all the more concerning that tinnitus symptom severity was so high higher in our veteran population.

\section{CONCLUSIONS}

There is a lack of research into the impact of tinnitus on the lived experience of (current and) ex-service personnel in the UK, with most research in such populations being conducted in the USA. Here we have taken a cross-disciplinary approach to interpreting the same dataset and consider ways forward. The findings of this survey confirm the need to further dedicated work to fully understand the impact tinnitus has, how impact can be meaningfully measured, what general support is needed and what are candidate therapeutic approaches that could improve the lives of UK veterans with tinnitus. In terms of the latter, and given veterans in the current study identified a lack of awareness and/ or understanding of tinnitus and the impact it can have on a person both functionally and emotionally, tailored interventions should be explored. The findings from this survey will immediately inform a series of semistructured interviews to be undertaken by the authors to triangulate our understanding of the lived experience and impact of tinnitus on UK veterans.

Contributors DJH and DS designed the study and secured project funding. DJH and GBOC designed survey and analysed survey data. DJH and GBOC drafted manuscript. DS reviewed manuscript and all authors approved final paper.

Funding This study was funded by The Royal British Legion, grant number: Aged Veterans Fund/TRBL09-BTA.

Competing interests None declared. 
Patient consent for publication Not required.

Provenance and peer review Not commissioned; externally peer reviewed.

Data availability statement Data are available on request from the authors.

Open access This is an open access article distributed in accordance with the Creative Commons Attribution Non Commercial (CC BY-NC 4.0) license, which permits others to distribute, remix, adapt, build upon this work non-commercially, and license their derivative works on different terms, provided the original work is properly cited, appropriate credit is given, any changes made indicated, and the use is non-commercial. See: http://creativecommons.org/licenses/by-nc/4.0/.

\section{NOTES}

1. Pawel J Jastreboff and Jonathan W. P. Hazell (2004). "Tinnitus Retraining Therapy: Implementing the Neurophysiological Model." Cambridge: Cambridge University Press.

2. Emily J. Watts et al. (2018). "Why is tinnitus a problem? A qualitative analysis of problems reported by tinnitus patients." Trends in hearing 22: $10.1177 / 2331216518812250$

3. Ryan D. Van Slyke, Nicholas J. Armstrong, and N. J Armstrong (2019). Communities Serve: A Systematic Review of Need Assessments on US Veteran and MilitaryConnected Populations. Armed Forces \& Society: 0095327×19845030.

4. E Fulton et al. (2019). "Transition from service to civvy street: the needs of armed forces veterans and their families in the UK." Perspectives in public health 139.1 (2019): 49-58.

5. Harriet Mellotte et al. (2017). Pathways into mental healthcare for UK veterans: a qualitative study, European Journal of Psychotraumatology, 8:1: $10.1080 / 20008198.2017 .1389207$

6. K Albertson (2017) "It's not just about recovery" The Right Turn Veteran-Specific Recovery Service Evaluation. https://www.fim-trust.org/wp-content/uploads/2017/06/ Albertson-et-al-Right-Turn-evaluation-final-report-June-2017-p.pdf

7. Celina H. Shirazipour et al. (2019). Psychosocial Aspects of Physical Activity Participation for Military Personnel with Illness and Injury: A Scoping Review. Military Behavioral Health: 1-18.

8. Christopher R Cederroth et al. (2019). Towards an Understanding of Tinnitus Heterogeneity. Frontiers in Aging Neuroscience:11, 53.

9. Emily J. Watts et al. (2018). "Why is tinnitus a problem? A qualitative analysis of problems reported by tinnitus patients." Trends in hearing 22. DOI: $10.1177 / 2331216518812250$

10. Helen Pryce et al. (2018). "Patient preferences in tinnitus outcomes and treatments: a qualitative study." International journal of audiology 57.10: 784-790.

11. Anaïs Tuepker et al. (2018). "A qualitative study of implementation and adaptations to Progressive Tinnitus Management (PTM) delivery." PloS one 13.5: e0196105.

12. The Royal British Legion (2014). "Lost Voices. A Royal British Legion report on hearing problems among Service personnel and veterans." London; The Royal British Legion.

13. D C Brown and R S Milner (2010). "A modern approach to noise induced hearing loss in military operations." Journal of the Royal Naval Medical Service 96, no. 1: 25.

14. Georgina Burns-O'Connell and Derek J. Hoare (2018). " Veterans, tinnitus, and research: A scoping review." Proceedings of the $15^{\text {th }}$ British Academy of Audiology annual conference, Liverpool, UK, 8-9 Nov (2018).

15. Jeremy Grimshaw (2014). "SURGE (The SUrvey Reporting GuidelinE)." Guidelines for Reporting Health Research: A User's Manual: 206-213.

16. Mary B Meikle et al. (2012). "The tinnitus functional index: development of a new clinical measure for chronic, intrusive tinnitus." Ear and hearing 33, no. 2: 153-176.

17. Kathryn Fackrell et al. (2016). "Psychometric properties of the Tinnitus Functional Index (TFI): Assessment in a UK research volunteer population." Hearing research 335: 220-235.

18. The Royal British Legion (2014). "Lost Voices. A Royal British Legion report on hearing problems among Service personnel and veterans." London; The Royal British Legion. https://www.rblcdn.co.uk/media/2282/lostvoiceshearinglossreport.pdf

19. Virginia Braun and Victoria Clarke (2006). "Using thematic analysis in psychology." Qualitative research in psychology 3, no. 2: 77-101.

20. Satu Elo and Helvi Kyngäs (2008). "The qualitative content analysis process." Journal of advanced nursing 62, 1: 107-115.

21. Virginia Braun and Victoria Clarke (2006). "Using thematic analysis in psychology." Qualitative research in psychology 3, no. 2: 77-101.

22. M Alvesson and K Sköldberg (2017). Reflexive methodology: New vistas for qualitative research. London, Sage.

23. S Kvale and S Brinkmann (2009). Interviews: Learning the craft of qualitative research interviewing, second Ed. London, Sage.

24. James A Henry et al. (2016). "Tinnitus Functional Index: Development, validation, outcomes research, and clinical application." Hearing research 334: 58-64.

25. Kathryn Fackrell et al. (2016). "Psychometric properties of the Tinnitus Functional
Index" 220-235.

26. James A Henry et al. (2015). "Validation of a novel combination hearing aid and tinnitus therapy device." Ear and Hearing 36, no. 1: 42-52.

27. Kathryn Fackrell et al. (2018). "Performance of the Tinnitus Functional Index as a diagnostic instrument in a UK clinical population." Hearing research 358: 74-85.

28. John S Phillips et al. (2018). "The natural history of subjective tinnitus in adults: A systematic review and meta-analysis of no-intervention periods in controlled trials." The Laryngoscope 128,1: 217-227. DOI: 10.1002/lary.26607

\section{BIBLIOGRAPHY}

Albertson, K. "It's not just about recovery. The Right Turn Veteran-Specific Recovery Service Evaluation.", 2017. https://www.fim-trust.org/wp-content/uploads/2017/06/ Albertson-et-al-Right-Turn-evaluation-final-report-June-2017-p.pdf.

Alvesson, M, and K Sköldberg. Reflexive methodology. London, Sage: New vistas for qualitative research, 2017.

Braun, Virginia, and Victoria Clarke. "Using thematic analysis in psychology." Qualitative Research in Psychology 3, no. 2 (2006): 77-101.

Brown, D C, and R S Milner. "A modern approach to noise induced hearing loss in military operations." Journal of the Royal Naval Medical Service 96, no. 1 (2010), 25.

Burns-O'Connell, Georgina, and Derek J. Hoare. "Veterans, tinnitus, and research: A scoping review. Proceedings of the 15 th British Academy of Audiology annual conference, Liverpool, UK." , 2018. https://www.tinnitus.org.uk/veterans-tinnitus-andresearch-a-scoping-review.

Cederroth, Christopher R, Silvano Gallus, Deborah A Hall, Tobias Kleinjung, Berthold Langguth, Antonello Maruotti, Martin Meyer., et al. "Editorial: towards an understanding of tinnitus heterogeneity." Frontiers in Aging Neuroscience 11 (2019), 53.

Elo, Satu, and Helvi Kyngäs. "The qualitative content analysis process." Journal of Advanced Nursing 62, no. 1 (2008): 107-15.

Erin, Martz. "A pilot randomized clinical trial comparing three brief group interventions for individuals with tinnitus." Global Advances in Health and Medicine 7, no. 10 (2018): 216495611878365

Fackrell, Kathryn, Deborah A Hall, Johanna G Barry, and Derek J Hoare. "Psychometric properties of the tinnitus functional index (TFI): assessment in a UK research volunteer population." Hearing Research 335 (2016): 220-35.

- Deborah A. Hall, Johanna G. Barry, and Derek J. Hoare. "Performance of the tinnitus functional index as a diagnostic instrument in a UK clinical population." Hearing Research 358 (2018): 74-85.

Forces in mind. "Call to mind: a framework for action.", 2015. http://www.fim-trust.org/ wp-content/uploads/2015/10/CALL-TO-MIND-REPORT.pdf.

Fulton, E, D Wild, J Hancock, E Fernandez, and J Linnane. "Transition from service to civvy street: the needs of armed forces veterans and their families in the UK." Perspectives in Public Health 139, no. 1 (2019): 49-58.

Goffman, Erving. The Presentation of Self in Everyday Life. Plymouth: The Penguin Press, 1969.

Grimshaw, Jeremy. "Surge (the survey reporting guideline)." Guidelines for Reporting Health Research: A User's Manual (2014): 206-13.

Henry, James A, Melissa Frederick, Sara Sell, Susan Griest, and Harvey Abrams. "Validation of a novel combination hearing aid and tinnitus therapy device." Ear and Hearing 36, no. 1 (2015): 42-52.

Henry, James A, Susan Griest, Emily Thielman, Garnett McMillan, Christine Kaelin, and Kathleen F Carlson. "Tinnitus functional index: development, validation, outcomes research, and clinical application." Hearing Research 334 (2016): 58-64.

Jastreboff, Pawel J, and Jonathan W. P. Hazell. Tinnitus Retraining Therapy: Implementing the Neurophysiological Model. Cambridge: Cambridge University Press, 2004.

Kvale, S, and S Brinkmann. Interviews: Learning the craft of qualitative research interviewing, 2 nd ed. London, Sage, 2009.

Meikle, Mary B, James A Henry, Susan E Griest, Barbara I Stewart, Harvey B Abrams, Rachel McArdle, Paula J Myers., et al. "The tinnitus functional index: development of a new clinical measure for chronic, intrusive tinnitus." Ear and hearing 33, no. 2 (2012): $153-76$.

Mellotte, Harriet, Dominic Murphy, Laura Rafferty, and Neil Greenberg. "Pathways into mental health care for UK veterans: a qualitative study." European Journal of Psychotraumatology 8, no. 1 (2017), 1.

Phillips, John S, Don J McFerran, Deborah A Hall, and Derek J Hoare. "The natural history of subjective tinnitus in adults: a systematic review and meta-analysis of no-intervention periods in controlled trials." The Laryngoscope 128, no. 1 (2018): 217-27.

Pryce, Helen, Amanda Hall, Rachel Shaw, Beth-Anne Culhane, Sarah Swift, Jean Straus, and Beth Claesen. "Patient preferences in tinnitus outcomes and treatments: a qualitative study." International Journal of Audiology 57, no. 10 (2018): 784-90.

Shirazipour, Celina H., Emily M. Tennant, Alice B. Aiken, and Amy E. Latimer-Cheung. "Psychosocial aspects of physical activity participation for military personnel with illness and injury: a scoping review." Military Behavioral Health 48, no. 2 (2019): $1-18$. 
The Royal British Legion. "Lost Voices. A Royal British Legion report on hearing problems among Service personnel and veterans. London; The Royal British Legion.", 2014. https://www.rblcdn.co.uk/media/2282/lostvoiceshearinglossreport. pdf.

Tuepker, Anaïs, Christine Elnitsky, Summer Newell, Tara Zaugg, and James A Henry. "A qualitative study of implementation and adaptations to progressive tinnitus management (PTM) delivery." Plos One 13, no. 5 (2018), e0196105.
Van Slyke, Ryan D., Nicholas J. Armstrong, and N. J Armstrong. "Communities serve: a systematic review of need assessments on U.S. veteran and Military-Connected populations." Armed Forces \& Society 16 (2019): $0095327 \times 1984503$.

Watts, Emily J., Kathryn Fackrell, Sandra Smith, Jacqueline Sheldrake, Haúla Haider, and Derek J. Hoare. "Why is tinnitus a problem? A qualitative analysis of problems reported by tinnitus patients." Trends in Hearing 22, no. 22 (2018): 233121651881225. 